\title{
Enhancing reliability of power supply systems with distributed generation plants
}

\author{
Bulatov Yuri ${ }^{1, *}$, Kryukov Andrey ${ }^{2}$ \\ ${ }^{1}$ Bratsk State University, Faculty of Power Engineering and Automation, Bratsk, Russia \\ ${ }^{2}$ Irkutsk State Transport University, Irkutsk National Research Technical University, Power Supply and Electrical Equipment \\ Department, Irkutsk, Russia
}

\begin{abstract}
The article present results of studies aimed at emergency control methods development in power supply systems equipped with distributed generation plants. The main purpose of emergency control is ensuring postemergency mode dead-beat stability and high quality of dynamic transitions for electrical network load-shedding. Control actions were formed by changing the vector of controlled parameters along a path corresponding to the shortest distance to the hypersurface of the limit modes. The dynamic processes quality when implementing the control actions was ensured based on the special settings of excitation and frequency automatic regulators. Based on computer-aided modelling, it is shown that the effective in-troduction of a postemergency mode to the stability region can be performed based on generalized equations of the limit loads while using the start algorithm ensuring mode's entering the stability region's neighboring boundary. The application of the fuzzy control algorithms for tuning regulators allows formation of a qualitative dynamic transition while load-shedding distributed generation plants in the postemergency mode.
\end{abstract}

\section{Introduction}

One of the goals of emergency control (EC) [1-3] systems application is to ensure the static stability of postemergency modes (PEM) of electrical power systems (EPS). The control actions in this case, are used to introduce the PEM into the permissible region (stability region) along a certain trajectory $D \mathbf{Y}$ in the space of the controlled parameters $\mathbf{Y}$. In practice, the trajectory $D \mathbf{Y}$ is assumed to be linear and can be defined proceeding from the following assumptions:

- to be set based on the preliminary calculations;

- to be determined based on the shortest distance to the limiting hypersurface [2], or by minimal damage related to cutting power sources and consumers.

Due to introduction of distributed generation (DG) plants in the immediate future, the modes introduction to the stability region becomes urgent for distribution networks and power supply grids and systems (PSS). It acquires special importance in networks equipped with DG plants, implemented on the basis of non-traditional renewable energy sources (NTRES) [4-9]. Such installations, as mini hydropower plants and offshore windmill farms can be removed from the consumption centers, which leads to a 'narrowing' of the areas of static dead-beat stability.

\section{Formulation of the problem}

The EC task is illustrated in Fig. 1, where the cross section of the stability region is shown with the coordinate plane of the active generating powers $P_{i}, P_{j}$ is shown; here and further in the text it is assumed that the stability and transmitted power limits coincide [2]. Curve 1 corresponds to the boundary of the stability region for the complete network diagram, curve 2 corresponds to a similar boundary when one of the main transmission lines is disconnected, and curve 3 corresponds to condition $\mathfrak{I}=$ const, where $\mathfrak{I}-$ the required value of the stability margin of the PEM.

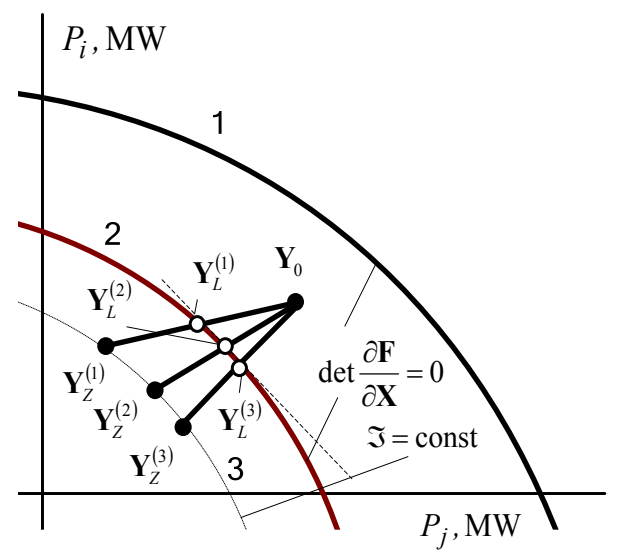

Fig. 1. To the sum of PEM introduction to the stability region: $\frac{\partial \mathbf{F}}{\partial \mathbf{X}}$ - matrix of Jacobi for steady-state equations (SSE); $\mathfrak{I}$ - stability reserve

The task of EC application is to reach one of the points $\mathbf{Y}_{Z}^{(1)}, \mathbf{Y}_{Z}^{(2)}, \mathbf{Y}_{Z}^{(3)}$ :

\footnotetext{
* Corresponding author: bulatovyura@yandex.ru
} 


$$
\begin{gathered}
\mathbf{Y}_{Z}^{(1)}=\mathbf{Y}_{0}+D \mathbf{Y}^{(1)}=\mathbf{Y}_{0}+t_{1} \Delta \mathbf{Y}^{(1)} ; \\
\mathbf{Y}_{Z}^{(2)}=\mathbf{Y}_{0}+D \mathbf{Y}^{(2)}=\mathbf{Y}_{0}+t_{2} \Delta \mathbf{Y}^{(2)} ; \\
\mathbf{Y}_{Z}^{(3)}=\mathbf{Y}_{0}+D \mathbf{Y}^{(3)}=\mathbf{Y}_{0}+t_{3} \Delta \mathbf{Y}^{(3)},
\end{gathered}
$$

where $D \mathbf{Y}^{(k)}, k=1 . .3$ - the trajectories of regime change in space $\mathbf{Y} ; \Delta \mathbf{Y}^{(k)}, k=1 . .3$ - direction of regime change; $t_{k}, k=1 . .3$ - scalar parameters that determine the amount of load-shedding in the relevant direction; index $k=1$ corresponds to direction set antecedently, index $k=2$ corresponds to load shedding the direction of the normal to the limiting hypersurface, while $k=3-$ to load shedding, ensuring minimal possible damage from cutoffs of power sources and consumers.

Load-shedding in the direction chosen $\Delta \mathbf{Y}^{(k)}$ should be effected for an acceptable quality of the dynamic processes, which can be achieved through the use of automatic excitation regulators (AER) and automatic regulator of rotor speed (ARRS) of DG plants synchronous generators. The relatively small power of DG plants and the low value of rotors inertia constant of their generators require taking into account the mutual influence of AER and ARRS when tuning them. It should also be noted, that optimal control requires adjusting the tuning of AER and ARRS when significant changes are introduced in the operation modes of both DG plants and PSS. These requirements can be met by using the intelligent control algorithms [10-15].

Below are the results of research aimed at developing methods for introduction of the mode into the stability region by the set trajectories, as well as algorithms for the functioning and adjusting of AER and ARRS.

\section{Introduction of a postemergency mode to the stability area}

The introduction of PEM to the stability region can be effected based on limit loads equations (LLE) [2]:

$$
\left.\begin{array}{l}
\mathbf{F}[\mathbf{X}, \mathbf{Y}(t)]=\mathbf{0} \\
\mathbf{V}[\mathbf{X}, \mathbf{R}, \mathbf{Y}(t)]=\left(\frac{\partial \mathbf{F}}{\partial \mathbf{X}}\right)^{T} \mathbf{R}=\mathbf{0} ; \\
U(\mathbf{R})=\mathbf{R}^{T} \mathbf{R}-1=0
\end{array}\right\}
$$

where $\mathbf{F}-\ell$ - dimensional function corresponding SSE; $\mathbf{X}-\ell-$ uncontrolled parameters dimensional vector; $\mathbf{Y}$ $m$ - controlled parameters dimensional vector; $\mathbf{Y}(t)=\mathbf{Y}_{0}+t \Delta \mathbf{Y} ; \quad \mathbf{R}-$ matrix own vector $\left(\frac{\partial \mathbf{F}}{\partial \mathbf{X}}\right)^{T}$, corresponding to zero eigenvalue.

Multiple calculation experiments [2] showed that on the basis of equations (1) the EPS regime can be introduced to the boundary of the stability region: the point $\mathbf{Y}_{L}^{(1)}$ (Fig. 1). To achieve a stability reserve necessary, an additional load shedding should be effected in direction $\Delta \mathbf{Y}^{(1)}$ (point $\mathbf{Y}_{Z}^{(1)}$ ) or in the direction of $\mathbf{R}$ vector, which coincides with the normal to the limiting hypersurface [2].
Though, in some cases the outreach to a 'distant boundary' of stability region [16] is possible: the point $\mathbf{Y}_{L}^{(d b)}$ in Fig. 2; in this case, the solution obtained differing in the nodal powers injections symbols reverse, cannot be used in practice.

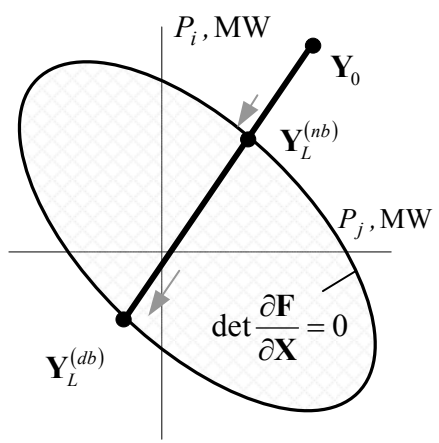

Fig. 2. To the problem of the 'distant boundary'

An effective and generalized way to solve the 'distant boundary' problem can be implemented based on starting algorithms using 'high order' methods [2, 17] or residual functional minimizing of the Steady-State Equations (SSE) [17]:

$$
\aleph(\mathbf{X})=\mathbf{F}^{T}\left(\mathbf{X}, \mathbf{Y}_{0}\right) \cdot \mathbf{F}\left(\mathbf{X}, \mathbf{Y}_{0}\right) .
$$

The use of $\mathbf{X}$ parameters as first approximations calculated as a result of functional (2) minimizing when solving limit loads equations (LLE) and their modifications, ensures a reliable convergence to the required points $\mathbf{Y}_{L}^{(n b)}$, found on the 'neighboring' boundaries of the stability region.

\section{The formation of quality dynamic transition when load-shedding generators}

Due to relatively small power of DG plants generators and their rotors low inertia constant, harmonized configuring of AER and ARRS becomes especially urgent. The harmonized configuring principle is defining optimal coefficients of AER and ARRS configuring ensuring minimal voltage and frequency deviations from the set values, high damping properties for electromechanical transition processes and the required stability reserve. Optimization of settings is performed based on genetic algorithm while using quadratic criterion $[14,15]$ :

$$
J=\int_{0}^{\Omega} e^{2}(j \omega) d \omega \rightarrow \min ,
$$

where $e(j \omega)=D^{D}(j \omega)-D^{M}(j \omega)$ - error between the desired and model sets of characteristic polynomials factors; $\omega$ - frequency current value from range $[0 ; \Omega]$ that determines the system 'bandwidth'. Butterworth or other polynomials can be used as desired polynomials.

The method of harmonized AER and ARRS configuring allows defining the optimal coefficients of regulators setting for different modes of PSS operation. For this purpose the use of auto-tuning unit is suggested 
with mode identification modules and regulators harmonized setting. Fig. 3 shows a block diagram of the suggested fuzzy control system. The input parameters are the current values of voltage $U_{g}$, rotor rotational frequency $\omega_{g}$ and DG plant power values $P_{g}, Q_{g}$. AER and ARRS tuning factors optimal for a current mode are determined based on the system performance. A more detailed description of the fuzzy control of AER and ARRS tuning is represented in works [14]. During the emergency control mode, the generators powers are varied in the postemergency mode $\left(\mathbf{Y}_{Z}\right.$ vector components) defined by the above method.

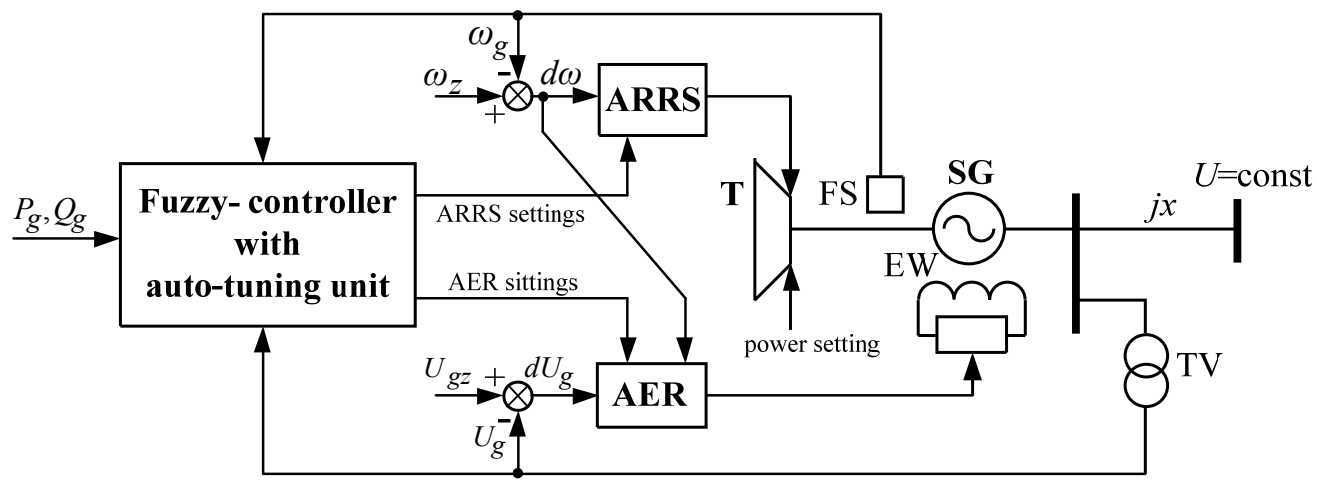

Fig. 3. Structural scheme of fuzzy system for control AER and ARRS of the DG plant:

FS - frequency sensor; EW - excitation winding; SG - synchronous generator; T - turbine; TV - voltage transformer

When identifying the mode based on experimental data, the closed-loop control system model for DG plants is formed. For this purpose, nonparametric identification is used, as a result of which, on the basis of a priori information about the process, the numerical values of the complex activation factor are determined as the ratio of the spectra of the output and input signals [18]. The 'turbine-generator' closed-loop system is demonstrated by the diagram shown in Fig. 4.

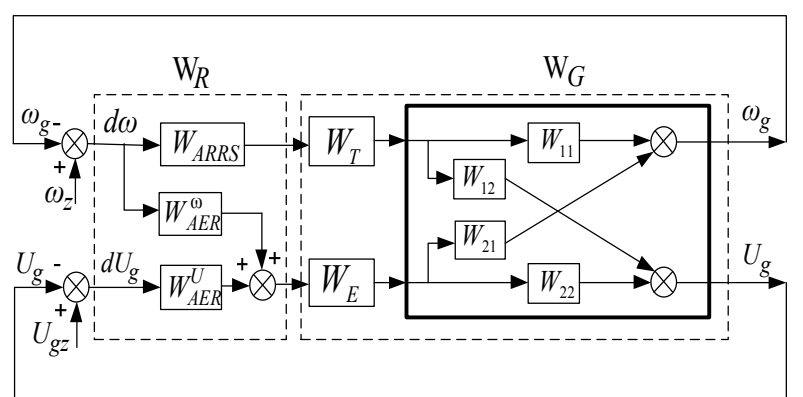

Fig. 4. The 'turbine-generator' system structural diagram: $W_{T}$ - complex turbine gear ratio; $W_{E}$ - complex exciter gear ratio

Characteristic polynomial of the system under consideration is determined using the expression:

$$
D^{M}(j \omega)=\operatorname{det}\left[\mathbf{E}+\mathbf{W}_{G}(j \omega) \cdot \mathbf{W}_{R}(j \omega)\right],
$$

where $\mathbf{E}$ - a single matrix; $\mathbf{W}_{G}(j \omega)$ - matrix activation function of the tunned object (of 'turbine-generator' system) determined experimentally; $\mathbf{W}_{R}(j \omega)-$ matrix activation function of the controller considering interconnection AER and ARRS: $\mathbf{W}_{R}(j \omega)=\left[\begin{array}{cc}W_{A R R S}(j \omega) & W_{A E R}^{\omega}(j \omega) \\ 0 & W_{A E R}^{U}(j \omega)\end{array}\right] ; W_{A R R S}(j \omega)-$ ARRS complex activation factor; $W_{A E R}^{\omega}(j \omega)$ - AER complex activation factor of the frequency-tuning channel; $W_{A E R}^{U}(j \omega)$ - AER complex activation factor of the voltage-tuning channel.

The following ratios can be recorded for activation factors:

$$
\begin{aligned}
& W_{\mathrm{ARRS}}(j \omega)=\left(k_{p}+\frac{k_{i}}{0,1 j \omega}+\frac{j k_{d} \omega}{j \omega+1}\right) \cdot \frac{1}{0,01 j \omega+1}, \\
& W_{\mathrm{AER}}^{\omega}(j \omega)=\frac{1+0,5 j \omega}{0,5 j \omega} \cdot\left[\frac{2 k_{0 \omega} j \omega}{(2 j \omega+1)(0,02 j \omega+1)}+\frac{0,05 k_{1 \omega} j \omega}{0,05 j \omega+1}\right], \\
& W_{\mathrm{AER}}^{\mathrm{U}}(j \omega)=\frac{1+0,5 j \omega}{0,5 j \omega} \cdot\left(k_{0 u}-\frac{0,02 k_{1 u} j \omega}{0,06 j \omega+1}\right),
\end{aligned}
$$

where $k_{p}, k_{i}, k_{d}$-ARRS tuning factors; $k_{0 u}, k_{1 u}, k_{0 \omega}$ and $k_{1 \omega}-$ AER adjusting channels tuning factors.

\section{The modelling results}

The point defining $\mathbf{Y}_{L}^{(1)}$ based on limit loads equations (LLE) (1) was carried out with regard to the PSS diagram showed in Fig. 5.

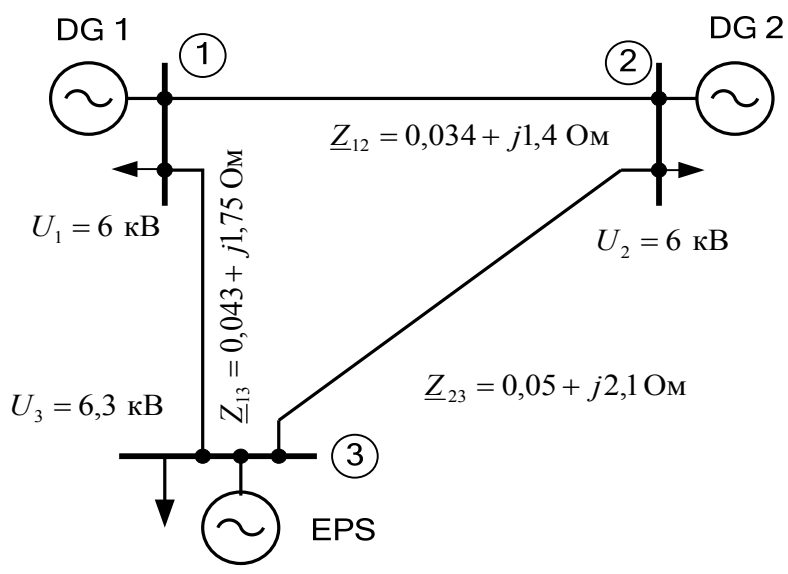

Fig. 5. Network diagram 
In this diagram, two mini hydropower plants with a power of $24 \mathrm{MW}$ each, operate at an industrial point load (timber processing facilities) connected at nodes 1 and 2 . Network voltage choice is determined by the presence of a large amount of high-voltage motors with $6 \mathrm{kV}$ voltage. The enterprises operate in one shift and in the hours of the evening maximum of the EPS, a power output of $15 \mathrm{MW}$ from each generator to the receiving system (node 3) is carried out. The network is implemented via flexible symmetrical electrical pathways [19]. As an emergency mode, the line 1-3 switching off was considered. One of the options for PEM entry into the stability region by the shortest trajectory on the basis of equations (1) is illustrated in Fig. 6.

The computer-aided modelling showed that when using LLE (1) and the starting algorithm, based on residual functional minimizing (2) a reliable outreach to the required boundary of the stability area is ensured.

In addition, the modelling was carried out in the MATLAB system, taking into account the AER and ARRS models. In this case, using the method of concordant setting, regulators parameters were identified for different modes and the base of fuzzy control system rules was generated.

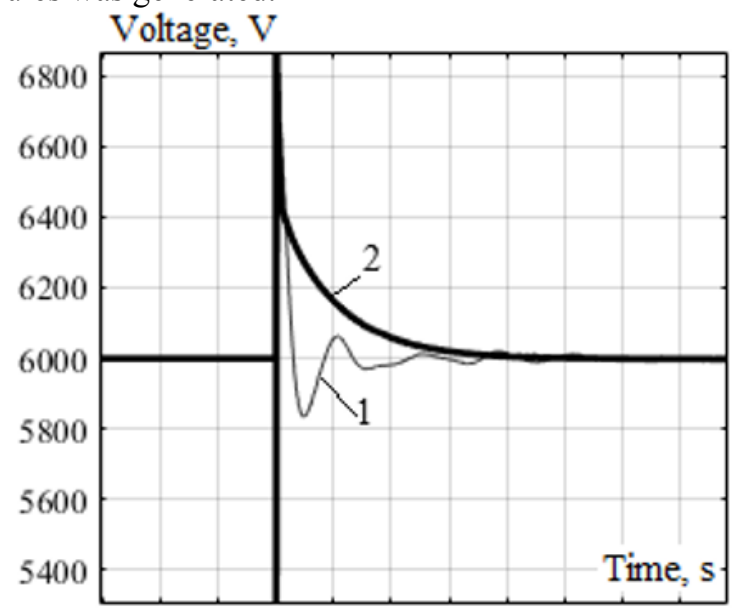

33.53434 .53535 .53636 .53737 .53838 .5

Rotor speed, r.u.

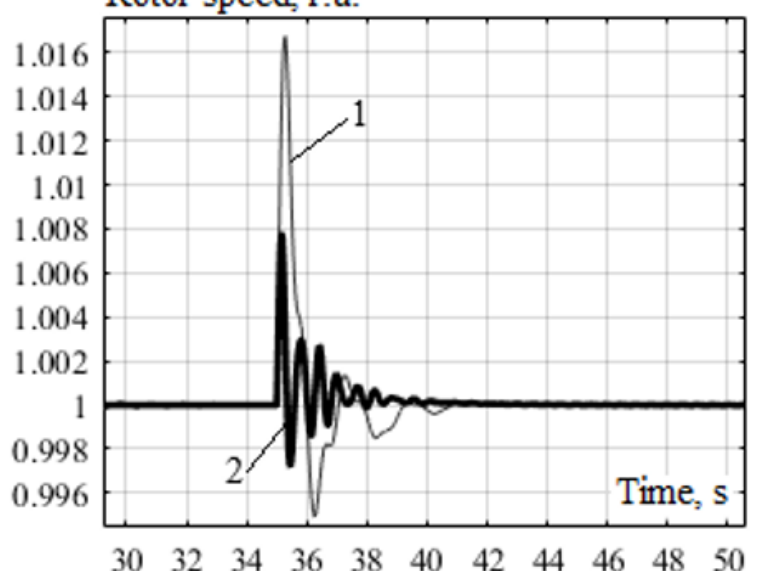

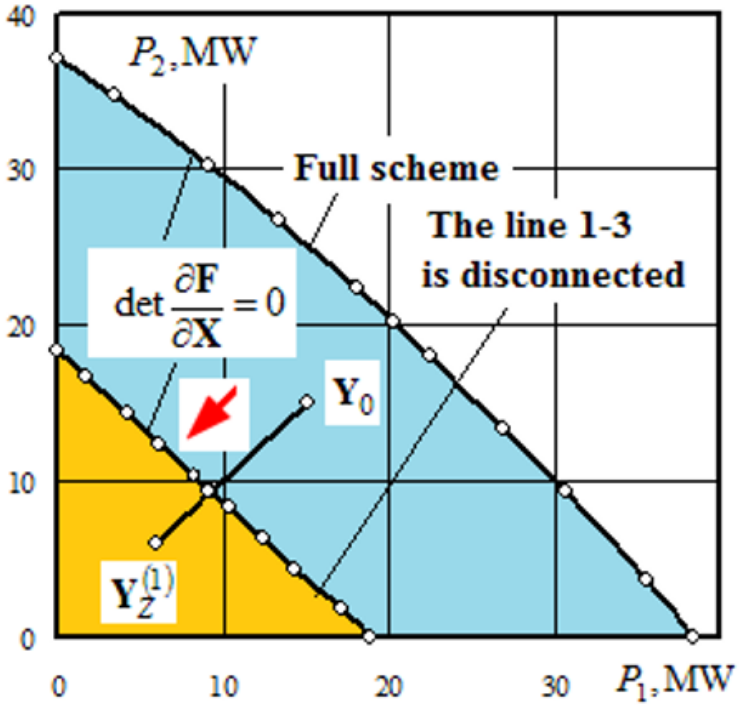

Fig. 6. Mode introduction into the stability area via the set trajectory while using equations (1): $\mathbf{Y}_{0}=\left[\begin{array}{ll}15 & 15\end{array}\right]^{T}$; $\mathbf{Y}_{Z}^{(1)}=\left[\begin{array}{ll}6 & 6\end{array}\right]^{T}$

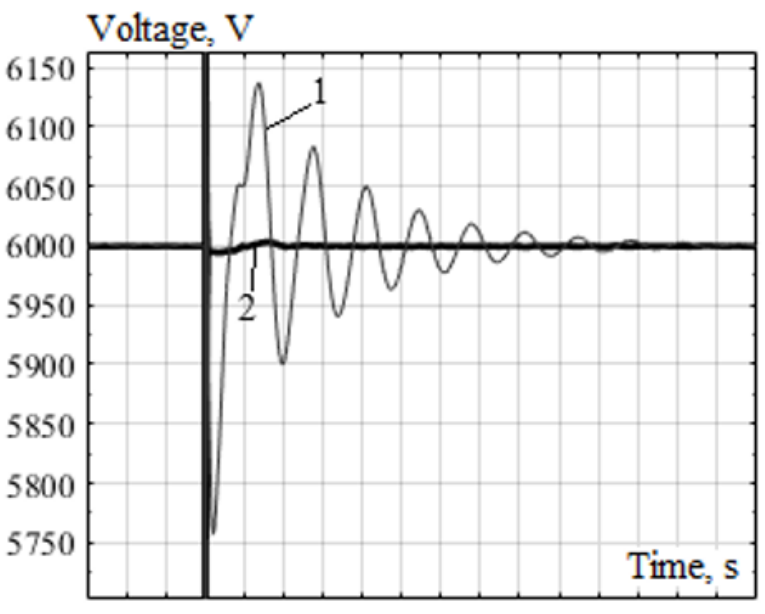

33343536373839404142434445464748

Rotor speed, r.u.

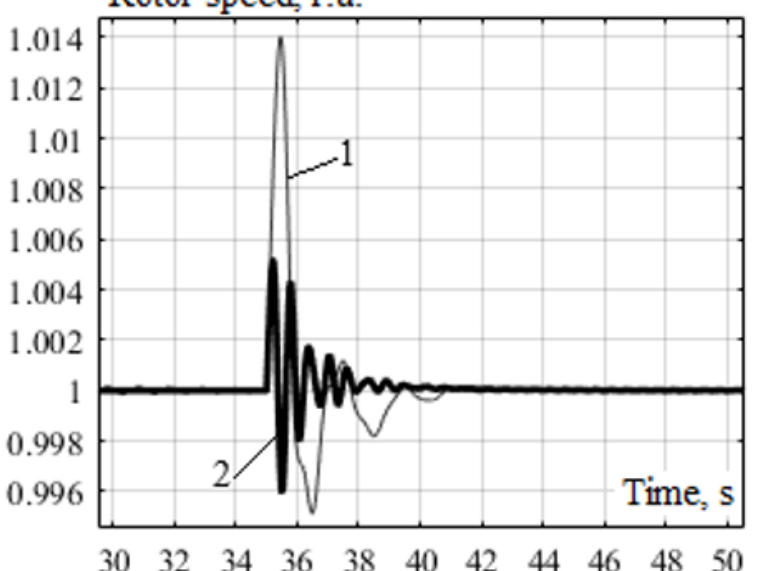


Active generator power, MW

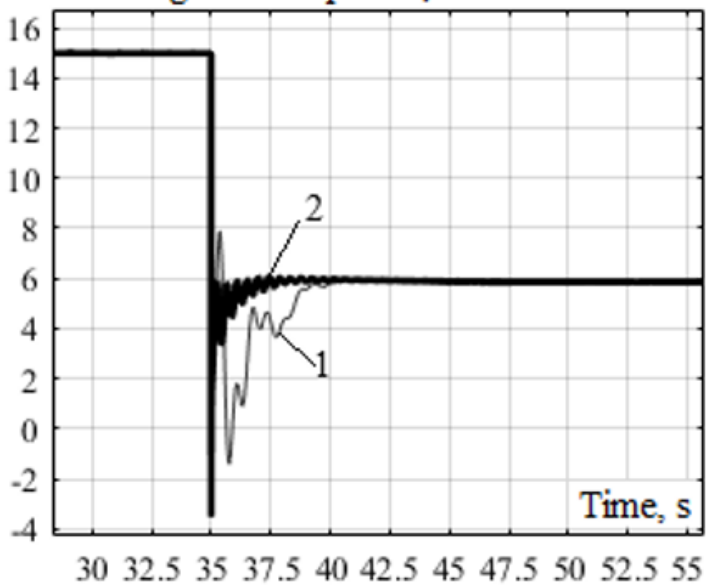

a) for DG1
Active generator power, MW

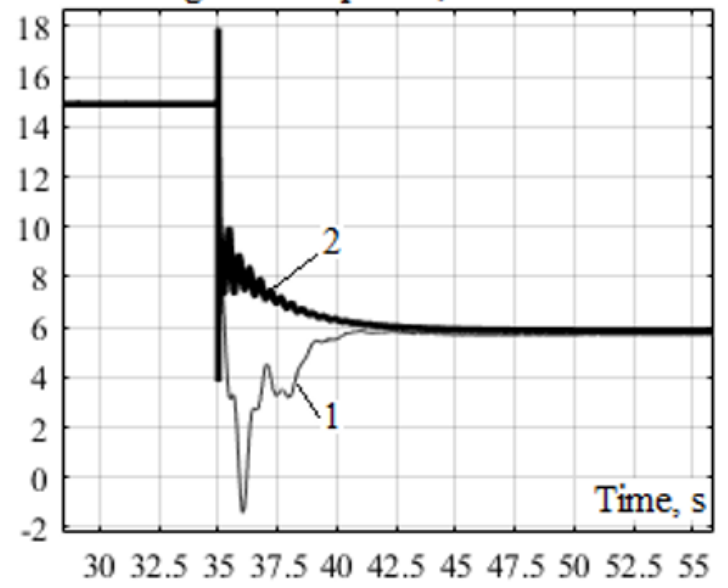

b) for DG2

Fig. 7. Oscillograms for voltage, frequency and power of DG plants when lines 1-3 are cut off: 1 - without change in AER and ARRS tuning factors; 2 - fuzzy control of AER and ARRS settings

When DG plants operate without regulators, the system loses stability, in case one of connections with infinite buses is cut off. To ensure PEM stability, DG plants generators should be load-shedded. In this case, the regulators harmonized tuning and its change in generators different operation modes allows to significantly enhance the transient processes quality parameters. The corresponding oscillograms of the voltage, frequency, and power of the DG plants when the lines 1-3 are cut off are shown in Fig. 7.

\section{Conclusion}

The following conclusions can be formulated based on the performed calculations and computer-aided modelling:

1. When DG plants operate without regulators, the stability of DG generators parallel operation is lost, in case one of connections is cut off from EPS receiving system.

2. Based on computer-aided modelling, it is shown that the effective introduction of a postemergency mode to the stability region can be performed based on generalized equations of the limit loads while using the start algorithm ensuring mode's entering the stability region's neighboring boundary.

3. The application of the fuzzy control algorithms for tuning AER and ARRS allows formation of a qualitative dynamic transition while load-shedding DG plants in the postemergency mode.

\section{References}

1. Innovative Power Engineering - 21 / Ed. V.M. Batenin, V.V. Bushuev, N.I. Voropai, Moscow: Information Center "Energia", 584 p. (2017)

2. A.V. Kryukov Limiting regimes of electric power systems, Irkutsk: IrGUPS, 236 p. (2016)

3. N.I. Voropai, V.G. Kurbatsky, N.V. Tomin [and others], Complex of intellectual means for prevention of major accidents in power systems, Novosibirsk: Science, 332 p. (2016)

4. N.I. Voropai, Z.A. Stychinsky, Renewable energy sources: theoretical foundations, technologies, technical characteristics, economics, Magdeburg: Otto-von-Guericke-Universität, 223 p. (2010)

5. O. Ellabban, H. Abu-Rub, F. Blaabjerg, Renewable energy resources: Current status, future prospects and their enabling technology, Renewable and Sustainable Energy Reviews, 39, pp. 748-764 (2014)

6. A.Q. Huang, M.L. Crow, G.T. Heydt, J.P. Zheng, The Future Renewable Electric Energy Delivery and Management (FREEDM) System: The Energy Internet, S. J. N. I. S. (2010). Proceedings of the IEEE. DOI: 10.1109/JPROC.2010.2081330

7. D. Egre and J.C Milewski, The diversity of hydropower projects, Energy Policy, 30 (14), pp. 1225-1230 (2002)

8. S.C.E. Jupe, A. Michiorri, P.C. Taylor, Increasing the energy yield of generation from new and renewable energy sources, Renewable energy, 14 (2), pp. 37-62 (2007)

9. Torriti J. Demand, Side Management for the European Supergrid: Occupancy variances of European single-person households, Energy Policy, 44, pp. 199-206 (2012)

10. K. Suslov, N. Solonina, V. Stepanov, A principle of power quality control in the intelligent distribution networks, International symposium on smart electric distribution systems and technologies EDST 2015 Proceedings, pp. 260-264 (2015)

11. Y. Chen, Y. Ma, W. Yun, Application of Improved Genetic Algorithm in PID Controller Parameters Optimization, Telkomnika, Vol. 11, No. 3, pp. 15241530 (2013)

12. A.Y. Jaen-Cuellar, R. de J. Romero-Troncoso, L. Morales-Velazquez, R.A. Osornio-Rios, PIDController Tuning Optimization with Genetic Algorithms in Servo Systems, International Journal 
of Advanced Robotic Systems, 10, pp. 324-337 (2013).

13. Yu.N. Bulatov, A.V. Kryukov, K.V. Suslov, Multiagent technologies for control of distributed generation plants in the isolated power systems, Far East Journal of Electronics and Communications, T. 17, № 5, pp. 1197-1212 (2017)

14. A.V. Kryukov, S.K. Kargapol'cev, Yu.N. Bulatov, O.N. Skrypnik, B.F. Kuznetsov, Intelligent control of the regulators adjustment of the distributed generation installation, Far East Journal of Electronics and Communications, T.17, № 5, pp. 1127-1140 (2017)

15. Yu.N. Bulatov, A.V. Kryukov, Optimization of automatic regulator settings of the distributed generation plants on the basis of genetic algorithm. 2nd International Conference on Industrial
Engineering, Applications and Manufacturing (ICIEAM). IEEE Conference Publications, pp. 1-6 (2016). doi: 10.1109/ICIEAM.2016.7911456

16. B.I. Ayuyev, V.V. Davydov, P.M. Erokhin, Optimization model of limiting modes of electrical systems, Electricity, 11, pp.3-11 (2010)

17. V.I. Tarasov, Theoretical bases of the analysis of the steady-state regimes of electric power systems, Novosibirsk: Science, 344 p. (2002)

18. Yu.N. Bulatov, A.V. Kryukov, Application of wavelet transform and genetic algorithms to tune automatic regulators of distributed generation plants, Sci. Bull. Novosibirsk State Tech. Univ., No. 2. T.63, pp. 7-22 (2016)

19. A.M. Semchinov, Current conductors of industrial enterprises, Energia, 200 p. (1972) 